\title{
The Coupling of Dynamics in Coupled Map Lattices
}

\author{
J.Y. CHEN ${ }^{\mathrm{a}, *}$, K.W. WONG ${ }^{\mathrm{a}}$, H.Y. ZHENG ${ }^{\mathrm{a}, \mathrm{b}}$ and J.W. SHUAI \\ ${ }^{\mathrm{a}}$ Department of Electronic Engineering, City University of Hong Kong, Tat Chee Avenue, Hong Kong, China; ${ }^{\mathrm{b}}$ Department of Electronic Engineering, \\ Xiamen University, Xiamen, China; ${ }^{\mathrm{c}}$ Department of Biomedical Engineering, Case Western Reserve University, Cleveland, OH 44106, USA
}

(Received 3 May 2001)

\begin{abstract}
We investigate the coupling of dynamics in coupled map lattices (CMLs) which is not only related to coupled parameter, but also the asynchronization among different mean fields in the lattices. Computer simulations show that the optimal coupling among mean fields can be found from the maximum coupling of dynamics in various CMLs. As a consequence, the application areas of coupled systems may be broadened due to the better understanding of their dynamics.
\end{abstract}

Keywords: Chaos; Coupled map lattices; Coupling of dynamics; Mean fields; Asynchronization

PACS numbers: $05.45 .+b$

Coupled chaotic systems are important in the research of chaos. Not only can they serve as models of nonlinear spatially extended systems, but they also play an important role in understanding the mechanism of biological information processing (Kaneko, 1989). Coupled map lattices (CMLs) are simple models of spatio-temporal chaos and have been extensively investigated by a number of researchers (Kaneko, 1995; Lemaitre and Chate, 1998; Belykh et al., 1998; Chen et al., 1998; 1999; 2000). They can be analyzed based on the collective movements of coupled oscillators (Willeboordse and Kaneko, 1994). In general, they have the form

$$
x_{n+1}(i)=(1-\varepsilon) f\left(x_{n}(i)\right)+\varepsilon h_{n}(i)
$$

where $x_{n}(i)$ is the $i$ th unit's value at time $n$; $(i=1,2, \ldots, M ; M$ is the size of the one-dimensional lattice) and $h_{n}(i)$ is the interaction mainly caused by other elements in the lattice. The first term at the right-hand-side of Eq. (1) represents the unit's dynamics given by a properly-selected nonlinear mapping function $f(x)$ while the second term is the coupling of the interaction through the coupled parameter $\varepsilon(0 \leq \varepsilon \leq 1)$.

In general, there are three types of coupling in CMLs. Those with local, direct-neighbor coupling have the form $h_{n}(i)=f\left(x_{n}(i+1)\right)$ or $h_{n}(i)=f\left(x_{n}(i-1)\right)$ are called oneway CMLs. They are simple coupled systems that are usually regarded as a kind of diffusion process. On the other hand, the interaction term of globally coupled maps is actually the mean field with the following form

$$
h_{n}(i)=H_{n}=\frac{1}{M} \sum_{j=1}^{M} f\left(x_{n}(j)\right) \quad \forall i=1,2, \ldots, M
$$

In general, the interaction term, $h_{n}(i)$ of intermediaterange coupling is given by

$$
h_{n}^{k}(i)=\frac{1}{2 K+1} \sum_{k=-K}^{K} f\left(x_{n}(i+k)\right)
$$

where $K$ is the coupling range, $1<K<M / 2$.

An application of chaotic systems is for communication (Pecora and Carroll, 1990; Sinha et al., 1992; Xiao et al., 1996; Rolf et al., 1998; Zhou and Lai, 1998; Anoniou et al., 1999; Chen et al., 2001). It is reported that a oneway CML with local, direct-neighbor coupling can be synchronized with another by just a scalar signal (Johnson et al., 1996; Peng et al., 1996). More complex coupled systems such as globally or intermediate coupled maps may have greater potential in secure communication as these systems can produce more complex high dimensional hyperchaos. In the investigation of various coupled systems, the coupled parameter $\varepsilon$ is frequently used to indicate the strength of coupling from other fields in the same lattice (Amengual et al.; Maistrenko et al., 1998). When $\varepsilon$ is small, it is weak coupling and the strength of coupling increases with $\varepsilon$. In different ranges of $\varepsilon$, there

*Corresponding author. E-mail: cjyok@yahoo.com 
are chaotic phenomena with different characteristics (Kaneko, 1995; Chen et al., 1998; 2000). From Eq. (1), it is obvious that $\varepsilon$ can represent the strength of coupling mainly contributed by other fields in the lattice. However, it cannot reflect the degree of asynchronization among the fields, which provides an implication on the coupling of dynamics in the coupled systems. We take the intermediate-range coupled system defined by Eqs. (1) and (3) as an example to illustrate this. The well-known logistic function $f(x)=1-\alpha x^{2}$ with $0 \leq \alpha \leq 2$ is used here as the mapping function. Let $M=101, K=40, \varepsilon=$ $0.8, \alpha=1.99$, and the initial field values are random numbers between 0 and 1 . After 1000 initial iterations, the lattice becomes a one-dimensional system with $x_{n}(1)=$ $x_{n}(2)=\ldots=x_{n}(101)$, i.e. all the field values are the same. The system enters a phase of global synchronization due to strong coupling among different fields. The corresponding synchronized motion can be chaotic or regular and the dynamics is as simple as that of a lattice formed by independent, uncoupled $(\varepsilon=0)$ elements (Heagy et al., 1994; Morelli and Zanette, 1998). In this case, the coupled parameter alone is not sufficient to indicate the actual coupling of the system dynamics as it just reflects a factor of the dynamics: the strength of coupling among different fields. The other factor, i.e., the degree of asynchronization among different fields should also be considered.

Based on the coupled parameter, we propose the coupling of dynamics to investigate the coupling of the dynamics in CMLs. It can be used in simple systems, as well as complex systems such as the intermediate-range coupled ones. In the following, we define the coupling of dynamics and present simulation results to show its properties.

The coupling of dynamics $\eta$ can be simply interpreted as follows

$$
\begin{gathered}
\eta=\varepsilon \rho \\
\rho=\frac{1}{N} \sum_{n=1}^{N} \Delta \bar{h}_{n}
\end{gathered}
$$

where

$$
\begin{gathered}
\Delta \bar{h}_{n}=\frac{1}{M} \sum_{i=1}^{M}\left(\bar{h}_{n}-h_{n}(i)\right)^{2} \\
\bar{h}_{n}=\frac{1}{M} \sum_{i=1}^{M} h_{n}(i)
\end{gathered}
$$

From Eq. (4), it is obvious that the coupling of dynamics is governed by two factors. One of them is the strength of coupling mainly caused by other fields in the lattice (i.e. the coupled parameter $\varepsilon$ ) while the other is the time-averaged mean-square deviation of the interaction $\rho$. The latter factor reflects the average degree of asynchronization among different fields from the very beginning to the current time $N$. In simple CMLs, the strongest coupling of dynamics is always achieved when $\varepsilon=1$. However, in complex CMLs such as those with intermediate-range coupling, too large of $\varepsilon$ may lead to a rapid decrease in $\rho$. Thus the strongest coupling of dynamics is not always achieved when $\varepsilon=1$. This is illustrated by the following two examples.

Example 1 The simplest type of locally-coupled CMLs, the one-way CML, is studied here. It is defined by the following equations

$$
\begin{gathered}
x_{n+1}(i)=\left(1-\varepsilon_{i}\right) f\left(x_{n}(i)\right)+\varepsilon_{i} f\left(x_{n}(i+1)\right) \\
x_{n}(M+1)=x_{n}(1)
\end{gathered}
$$

Here we choose logistic function in the lattice with size $M=101$ and $\varepsilon_{i}=\varepsilon \forall i=1,2, \ldots, M$. The range of $\varepsilon$ is from 0 to 1 and the precision of calculation is $\Delta \varepsilon=0.01$. After $N=20,000$ iterations, $\rho$ and $\eta$ are calculated with the first 1000 initial transients omitted. The results are plotted in Fig. 1a,b while the two largest Lyapunov exponents (Eckmann and Ruell, 1985) $\lambda_{\max 1}$ and $\lambda_{\max 2}$ $\left(\lambda_{\max 1}>\lambda_{\max 2}\right)$ are shown in Fig. 1c. From Fig. 1a, we find that $\rho$ does not always decrease monotonously with $\varepsilon$. When $\varepsilon=1$, both $\eta$ and $\rho$ are the strongest. The coupling of dynamics is thus the largest. In this simple coupled system, the strongest coupling of dynamics can also be found by using the coupled parameter $\varepsilon$. Note that $\eta$ increases with $\varepsilon$ although the graph in Fig. 1b is not strictly linear. This shows that the coupled parameter can also reflect the coupling of the dynamics, as the one-way CML is a simple coupled system. Figure 1c shows that there is only a small difference between the two largest Lyapunov exponents and thus leads to hyperchaos with various $\varepsilon$.

Example 2 We simulate the intermediate-range coupled system defined by Eqs. (1) and (3). The mapping function is the logistic function and the lattice size is $M=101$. In particular, $K$ varies between 1 and 50 and $\varepsilon$ is between 0 and 1. The precision of calculation is $\Delta K=1$ and $\Delta \varepsilon=$ 0.01 while the total number of iterations is $N=20,000$. After the 1000 initial transients are omitted, the maximum coupling of dynamics $\eta_{\max }$ and the corresponding value of

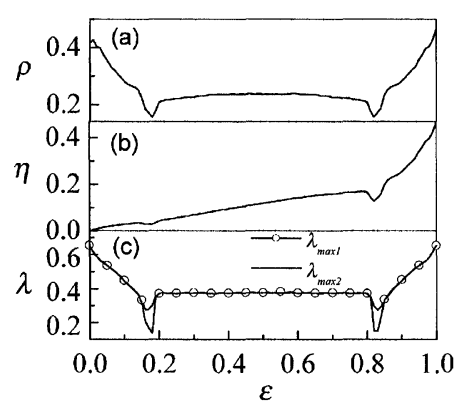

FIGURE 1 Characterization of the coupling of dynamics and Lyapunov exponents for various coupled parameter when $M=101$. (a) The degree of asynchronization among mean fields $\rho$, (b) the coupling of dynamics $\eta$, and (c) the two largest Lyapunov exponents $\lambda_{\max 1}$ and $\lambda_{\max 2}\left(\lambda_{\max 1}>\right.$ $\lambda_{\max 2}$ ) versus coupled parameter $\varepsilon$. 


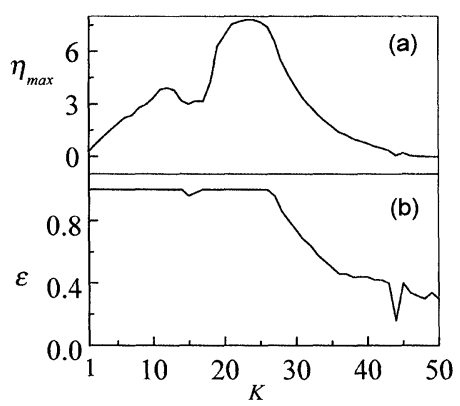

FIGURE 2 Characterization of the strongest coupling of dynamics for various coupled range $K$. (a) The strongest coupling of dynamics $\eta_{\max }$ versus $K$, and (b) the coupled parameter $\varepsilon$ versus $K$ when the coupling of dynamics is the strongest.

$\varepsilon$ are found for each $K$. The results are plotted in Fig. 2a, b. From the figures, we observe that with the increasing of $K$ from 1 to $25, \eta_{\max }$ increases accordingly (except a small drop in the region around $K=15$ ). When $K \approx 25$, the coupling of dynamics has the largest value among all the coupled ranges. At this point, the two factors $\varepsilon$ and $\rho$ achieve the best combination. The corresponding attractors are widely distributed in space. For the globally coupled case $(K=50), \eta_{\max }=0.002$ which corresponds to a weak coupling of dynamics. The system is similar to a one-dimensional logistic map that generates simple dynamics and locally distributed attractors. Evidently, this system is not suitable for some applications such as secure communication. From Fig. 2b, we observe that when $K$ is small, $\varepsilon$ is found to have the value around one. This shows that both the coupled parameter and the coupling dynamics possess the largest value at the same time. Within this range of intermediate coupling, the coupled parameter, together with the Lyapunov exponents are sufficient to reflect the dynamics of simple CMLs. However, when the coupling range is extended and the CMLs become more complex, these parameters cannot fully describe the system dynamics besides pointing out that a particular region in phase space is the coherent phase (i.e. $\eta=0$ ). Although the fully synchronization among different fields (corresponds to the case $\rho=0$ ) can easily be found from the field values during the iterations, the abandon of $\rho$ at regions where $\rho \neq 0$ may lead to a wrong analysis on the dynamics which may then affect the applications of CMLs.

Figure 3 is a plot of the coupling of dynamics and the Lyapunov exponents of the intermediate coupled system with $K=40$ versus the coupled parameter. In Fig. 3a, we observe that $\eta$ is at its maximum (0.6) when $\varepsilon=0.42$. At the point $\varepsilon=0$ and within the range $0.62<\varepsilon \leq 1, \eta=0$ which correspond to uncoupled and synchronized dynamics, respectively. Figure $3 \mathrm{~b}$ is a plot of the two largest Lyapunov exponents $\lambda_{\max 1}$ and $\lambda_{\max 2}\left(\lambda_{\max 1}>\right.$ $\lambda_{\max 2}$ ) versus $\varepsilon$. When $\eta=0, \lambda_{\max 1} \cong 0.66$ which is the value of the Lyapunov exponent in one-dimensional system. After omitted the 1000 initial transients, the fields $x_{n}(1), x_{n}(2), \ldots, x_{n}(101)$ have different styles at various coupled parameters. When $\varepsilon=0$, the fields are fully

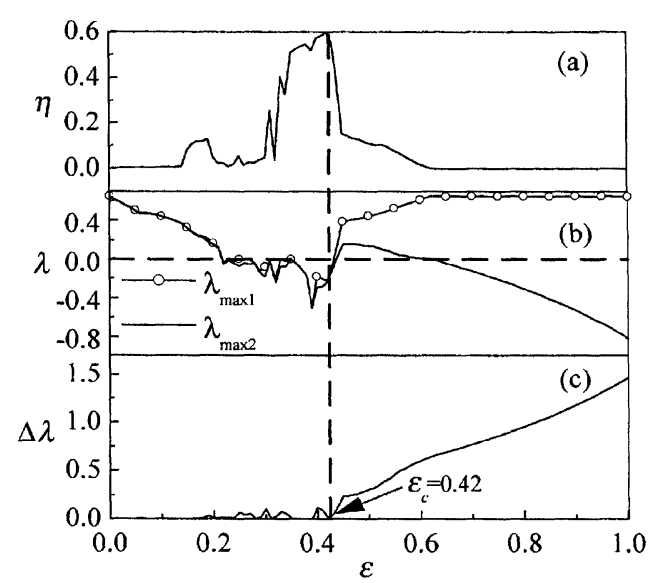

FIGURE 3 Properties of the coupling of dynamics and the Lyapunov exponents for various coupled parameter when $M=101$ and $K=40$. (a) The coupling of dynamics $\eta$, (b) the two largest Lyapunov exponents $\lambda_{\max 1}$ and $\lambda_{\max 2}\left(\lambda_{\max 1}>\lambda_{\max 2}\right)$ and (c) the difference in Lyaponov exponents $\Delta \lambda$ versus coupled parameter $\varepsilon$.

uncoupled and they lead to the highest value of $\rho$. When $0.62<\varepsilon \leq 1$, they are fully synchronized due to the strong coupling among different fields. The coupled parameter has different values in these two cases, but the corresponding coupling of dynamics are both zero. This implies that both dynamics are the same as that of a onedimensional map (Maistrenko et al., 1998). The difference of the two largest Lyapunov exponents $\Delta \lambda(\Delta \lambda=$ $\left.\lambda_{\max 1}-\lambda_{\max 2}\right)$ versus $\varepsilon$ is plotted in Fig. 3c. When $\varepsilon<$ $0.42, \Delta \lambda$ remains at small values. But when $\varepsilon>0.42, \Delta \lambda$ increases rapidly. As a result, $\varepsilon=0.42$ can be considered as a threshold $\varepsilon_{\mathrm{c}}$. At this point, the it has its largest value $\eta_{\max }=0.60$ which implies the strongest coupling of dynamics.

The proposed coupling of dynamics can reflect the actual coupling of the dynamics in various CMLs effectively and may advance their potential applications. For example, there are chaotic, as well as regular states in Figs. 1 and 3. In chaotic states, the large values of coupling of dynamics indicate low mutual correlation among different fields which is important in multi-channel spread-spectrum communication (Xiao et al., 1996). When $\varepsilon<\varepsilon_{c}$ (where $\varepsilon_{c}$ corresponds to the coupled parameter that leads to the largest coupling of dynamics. In Fig. $1, \varepsilon_{c}=1$ ), the chaotic states are hyperchaos because the difference among the two largest Lyapunov exponents are small. On the other hand, when $\varepsilon>\varepsilon_{c}$, the coupling of dynamics drops substantially and hyperchaos disappear no matter how large the lattice size is. In regular states, one of the important applications is for information processing in artificial intelligence. This application requires that the information representation is stable over a wide range of parameters. This means that the periods are relatively independent of the parameters (Ishii et al., 1996). If the coupling of dynamics can be kept at approximately the same value over a wide range of parameter, the intermediate coupled system with the corresponding range of parameter can meet the 
requirement. In Fig. 3a, when $0.35<\varepsilon<0.43$, the values of $\eta$ range are between 0.5 and 0.6 and it is suitable for information-processing applications.

To summarize, we have investigated the coupling of dynamics in both simple and complex CMLs developed from mapping functions. Simulation results show that for this purpose, it can reflect the properties of dynamics more effectively than the coupled parameter. Moreover, it leads to a deeper understanding of various coupled systems and may advance their applications.

\section{Acknowledgements}

The work described in this paper was fully supported by a Grant provided by City University of Hong Kong. Project No. 7001077.

\section{References}

Amengual, A., Hernandez, G.E., Montage, R., Miguel, M.S. (1997) "Synchronization of spatiotemporal chaos: the regime of coupled spatiotemporal intermittency", Physical Review Letters 78, $4379-4382$

Anoniou, I., Bosco, F., Ivanov, V.V. and Kisel, I.V. (1999) "Emergence of collective behavior in a system of autonomous agents", Discrete Dynamics in Nature and Society 3, 15-23.

Belykh, V., Belykh, I., Komrakov, N. and Mosekilde, E. (1998) "Invariant manifolds and cluster synchronization in a family of locally coupled map lattices", Discrete Dynamics in Nature and Society 4, 245-256.

Chen, J.Y., Chen, Z.X., Shuai, J.W., Lu, Y.Y. and Guo, D.H. (1998) "A chaotic lattice and the circuit of its unit", Canadian Journal of Physics 76, 801-808.

Chen, J.Y., Wong, K.W. and Shuai, J.W. (1999) "Finding the chaotic synchronizing state with gradient descent algorithm", Physics Letters A 263, 315-322.

Chen, J.Y., Wong, K.W., Chen, Z.X., Xu, S.C. and Shuai, J.W. (2000) "Phase synchronization in discrete chaotic systems", Physical Review E 61, 2559-2562.
Chen, J.Y., Wong, K.W., Zheng, H.Y. and Shuai, J.W. (2001) "Synchronizing spatiotemporal chaos by introducing a finite flat region in the local map", Discrete Dynamics in Nature and Society, $\mathbf{6}$, 201-206.

Eckmann, J.P. and Ruell, D. (1985) "Ergodic theory of chaos and strange attractors", Review Modem Physics 57, 617-656.

Heagy, J.F., Carrol, T.L. and Pecora, L.M. (1994) "Synchronous chaos in coupled oscillator systems", Physical Review E 50, 1874-1885.

Ishii, S., Fukumizu, K. and Watanabe, S. (1996) "A network of chaotic elements for information processing", Neural Networks 9, 25-40.

Johnson, G.A., Mar, D.J., Carroll, T.L. and Pecora, L.M. (1998) "Synchronization and imposed bifurcations in presence of large parameter mismatch", Physical Review Letters 80, 3956-3959.

Kaneko, K. (1989) "Chaotic but regular posi-nega switch among coded attractors by cluster-size variation", Physical Review Letters 63, 219-223.

Kaneko, K. (1995) "Remarks on the mean field dynamics of networks of chaotic elements", Physica D 86, 158-170.

Lemaitre, A. and Chate, H. (1998) "Nonperturbative renormalization group for chaotic coupled map lattices", Physical Review Letters 80 , $5528-5531$.

Maistrenko, Y.L., Maistrenko, V.L., Popovich, A. and Mosekilde, E. (1998) "Role of the absorbing area in chaotic synchronization", Physical Review Letters 80, 1638-1641.

Morelli, L.G. and Zanette, D.H. (1998) "Synchronization of stochastically coupled cellular automata", Physical Review E 58, 8-11.

Pecora, L.M. and Carroll, T.L. (1990) "Synchronization in chaotic systems", Physical Review Letters 64, 821-824.

Peng, J.H., Ding, E.J., Ding, M. and Yang, W. (1996) "Synchronizing hyperchaos with a scalar transmitted signal", Physical Review Letters 76, 904-907.

Rolf, J., Bohr, T. and Jensen, M.H. (1998) "Directed percolation universality in asynchronous evolution of spatiotemporal intermittency", Physical Review E 57, 2503-2506.

Sinha, S., Biswas, D., Azam, M. and Lawande, S.V. (1992) "Local-toglobal coupling in chaotic maps", Physical Review A 46, 6242-6246.

Willeboordse, F.H. and Kaneko, K. (1994) "Bifurcations and spatial chaos in an open flow model", Physical Review Letters 73, 533-536.

Xiao, J.H., Gu, G. and Qu, Z.L. (1996) "Synchronization of spatiotemporal chaos and its application to multichannel spreadspectrum communication", Physical Review Letters 77, 4162-4165.

Zhou, C.S. and Lai, C.H. (1998) "Synchronization with positive conditional lyapunov exponents”, Physical Review E 58, 5188-5191. 


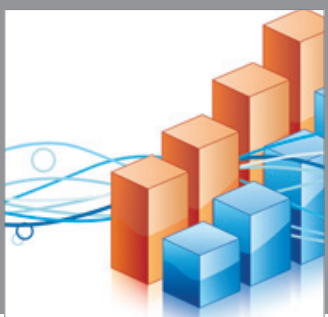

Advances in

Operations Research

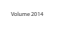

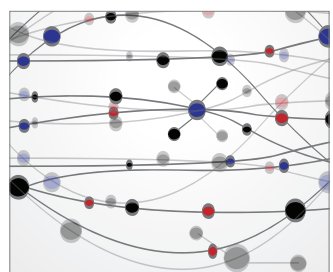

\section{The Scientific} World Journal
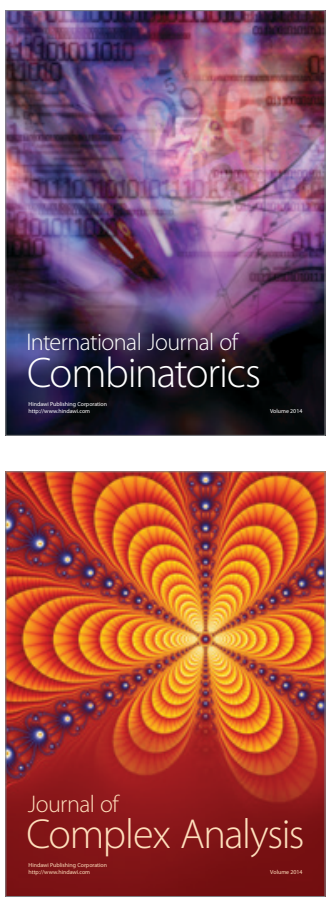

International Journal of

Mathematics and

Mathematical

Sciences
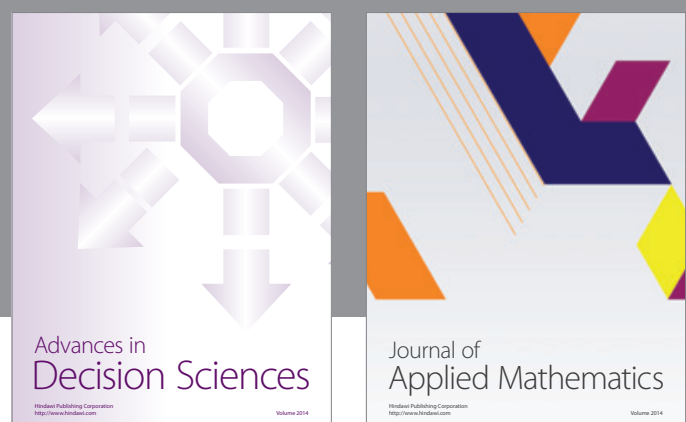

Journal of

Applied Mathematics
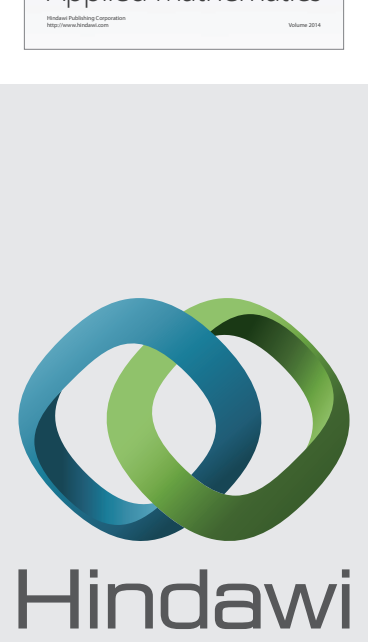

Submit your manuscripts at http://www.hindawi.com
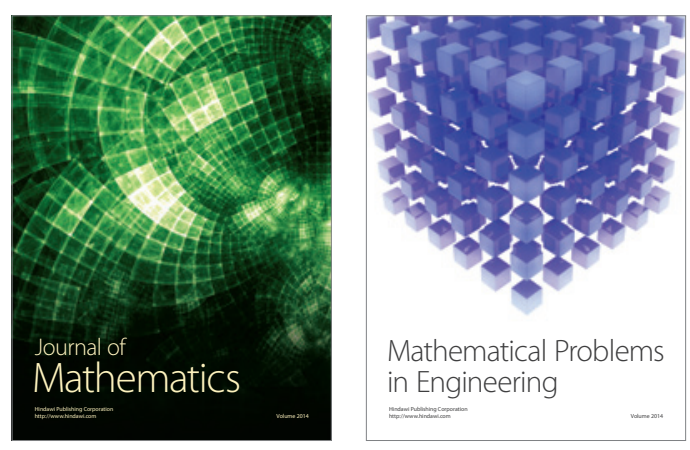

Mathematical Problems in Engineering
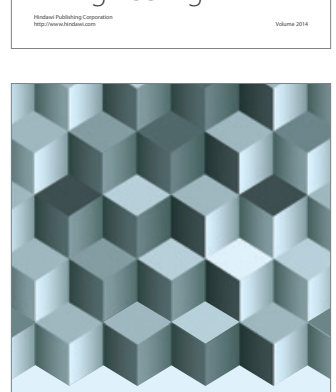

Journal of

Function Spaces
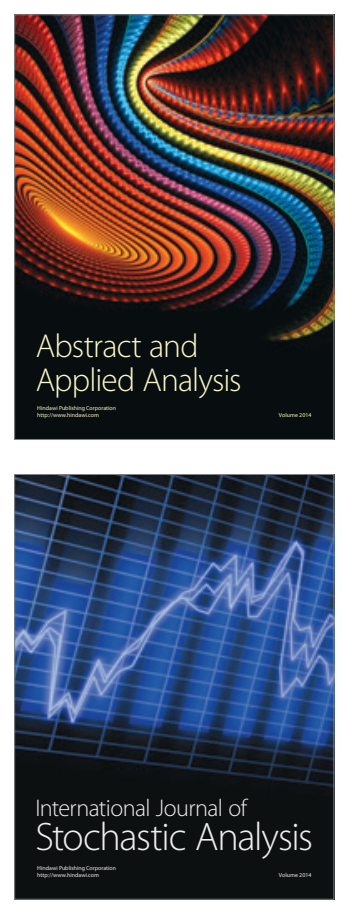

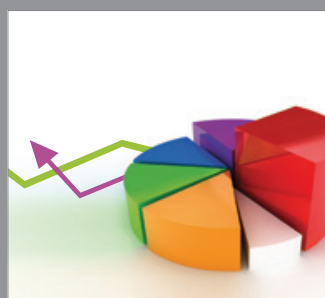

ournal of

Probability and Statistics

Promensencen
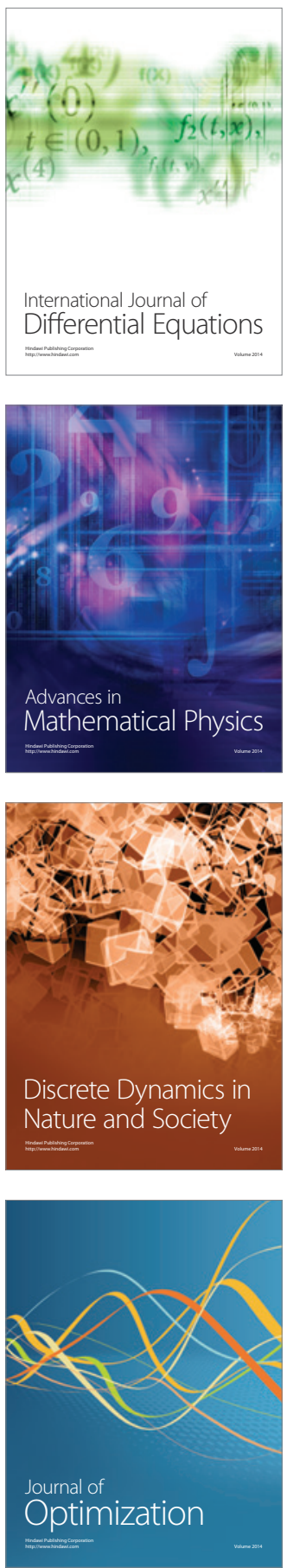